\title{
EVALUACIÓN DE DOS PROGRAMAS DE VACUNACIÓN CON LA CEPA 2512 DE LA ENFERMEDAD DE GUMBORO FRENTE A LA INFECCIÓN EXPERIMENTAL CON LA CEPA F52/70
}

\author{
Evaluation of Two Vaccination Programs Using the Infectious Bursal \\ Disease 2512 Strain Against Experimental Infection with the F52/70 Strain
}

Claudia Pérez C. ${ }^{1}$, Mónica Alba Ch. ${ }^{1,2}$ y Eliana Icochea D. ${ }^{1,3}$

\section{RESUMEN}

\begin{abstract}
El objetivo del presente estudio fue evaluar la inmunoprotección conferida por dos programas de vacunación en pollos de carne, con diferentes vacunas comerciales del virus de la Enfermedad Infecciosa de la Bursa (EIB). El grupo A fue vacunado con la cepa 2512 (intermedia-fuerte) a los 19 días de edad; el grupo B fue vacunado a los 10 días con la cepa 2512 (intermedia-intermedia) y a los 19 días con la cepa 228TC (intermedia-intermedia); y el grupo $\mathrm{C}$ fue el control no vacunado. Los tres grupos fueron desafiados a los 32 días de edad con la cepa estándar F52/70 de la EIB. La protección fue medida a través de signos clínicos (diarrea), tamaño de la bursa, índice bursal, lesiones histopatológicas en bursa, parámetros productivos y títulos de anticuerpos. El tamaño e índice bursal al $7^{\circ}$ día del desafío en los 3 grupos fueron compatibles con atrofia bursal, aunque no afectaron mayormente los parámetros productivos. El peso corporal y los títulos de anticuerpos al final del estudio fueron similares en los grupos A y B, no existiendo diferencia estadística significativa $(p>0.05)$ entre ellos. Los resultados obtenidos de las lesiones bursales, histopatología, índice bursal, parámetros productivos y títulos de anticuerpos indicaron que tanto las aves del grupo A como las del grupo B estuvieron protegidas contra el virus de desafío.
\end{abstract}

Palabras clave: Enfermedad infecciosa de la bursa, 2512 intermedia-fuerte, 2512 intermedia-intermedia, 228TC, índice bursal, histopatología

\section{Abstract}

The objective of the present study was to evaluate the immunoprotection given by two vaccination programs in broilers, using comercial vaccines against the infectious bursal disease virus (IBDV). In group A, the strain 2512 (intermediate plus) was inoculated at 19 days of age; in group B was used two intermediate-intermediate vaccines: the strain 2512 at day 10 and the strain $228 \mathrm{TC}$ at day 19; and group $\mathrm{C}$ was used as the nonvaccinated control group. The three groups were challenged at day 32 with the standard

\footnotetext{
${ }^{1}$ Laboratorio de Patología Aviar, Facultad de Medicina Veterinaria, Universidad Nacional Mayor de San Marcos, Lima

${ }^{2}$ Dirección actual: San Fernando S.A. E-mail: alba.monica@gmail.com

${ }^{3}$ E-mail: eliana.icochea@gmail.com
} 
strain F52/70 of IBDV. The protection was measured after the challenge through clinical signs (diarrhea), size of the bursa, bursal index, productive parameters and antibody titers. The size and the bursal index at 7 days post challenge in the three groups showed bursal atrophy but this, did not significantly affect productive parameters. Groups A and B had similar body weights and antibody titers at day 49 ( $p>0.05)$. The results obtained in bursal damage, histopathology, bursal index, productive parameters, and antibody titers indicated that chicks of groups A and B were protected against the IBDV strain of challenge.

Key words: Infectious Bursal Disease, 2512 intermediate plus, 2512 intermediateintermediate, 228TC, bursal index, histopathology

\section{INTRODUCCIÓN}

La Enfermedad de Gumboro o Enfermedad Infecciosa de la Bursa (EIB) es una enfermedad viral aguda y altamente contagiosa en aves jóvenes, de distribución mundial y que ha sido reconocida como una de las enfermedades más importantes en pollos (Saif, 2003). Se caracteriza por la destrucción de linfocitos B inmaduros en la bursa de Fabricio. Se inicia con un proceso inflamatorio y culmina con atrofia induciendo un estado severo de inmunosupresión (Banda y Villegas, 2002). La enfermedad tiene dos formas de presentación: la subclínica, cuando la enfermedad ocurre en aves menores de 3 semanas, y la clínica en aves de 3 a 6 semanas de edad.

La prevención y control de la enfermedad se hace, principalmente, a través de la vacunación. En el mercado, las vacunas comerciales más usadas son las que contienen cepas vacunales intermedias del virus de Gumboro, las cuales varían en antigenicidad y virulencia. Estas pueden producir mayor o menor grado de inmunosupresión debido a la atrofia de la bolsa de Fabricio, tejido donde se replica el virus (Guerrero, 2001).

La importancia económica de esta enfermedad está relacionada con la inmunosupresión que reduce la respuesta de anticuerpos a otras vacunas, incrementando la susceptibilidad a infecciones secundarias. Los efectos sobre el rendimiento productivo incluyen incremento en la mortalidad, reducción en la eficiencia alimenticia, baja de peso corporal e incremento en el porcentaje de decomisos (Hafez, 2002). En el país se ha venido presentando problemas sanitarios asociados a inmunosupresión y es posible que el virus de la Infección Bursal esté involucrado, por lo que se requiere nuevas alternativas para el control de esta enfermedad. Se tiene diferentes programas de vacunación y el uso de vacunas intermedias-fuertes, como algunas que contienen la cepa 2512 que ha sido considerada como una buena opción para la prevención y control de la enfermedad clínica. El presente estudio tuvo como objetivo evaluar la protección que confieren los programas vacunales que contienen la cepa 2512 del virus de Gumboro frente a la infección experimental con la cepa estándar F52/70 de la enfermedad infecciosa de la bursa.

\section{Materiales y Métodos}

\section{Lugar de Estudio}

Galpón experimental e instalaciones del Laboratorio de Patología Aviar y el Laboratorio de Histología, Embriología y Patología Veterinaria de la Facultad de Medicina Veterinaria de la Universidad Nacional Mayor de San Marcos, Lima. 


\begin{abstract}
Animales
Se emplearon 321 pollos de carne, machos, de un día de edad, de la línea Ross, que fueron distribuídos uniformemente en 3 grupos experimentales y criados en ambientes separados. Los pollos recibieron el mismo tipo de alimento y agua ad libitum. Se emplearon dos programas de vacunación a virus vivo contra la enfermedad infecciosa de la bursa: el grupo A fue vacunado con la cepa 2512 (intermedia-fuerte) a los 19 días de edad, el grupo B fue vacunado con la cepa 2512 (intermedia-intermedia) a los 10 días de edad y revacunado a los 19 días de edad con la cepa 228TC (intermedia-intermedia), y el grupo C quedó como control no vacunado. Al día 32 de edad se colocó a todos los pollos en un mismo ambiente, donde fueron desafiados con la cepa estándar F52/70, vía ocular.
\end{abstract}

\section{Evaluación de la Bursa}

El Índice Bursal (IB) se determinó mediante la siguiente fórmula:

$$
\begin{aligned}
\mathrm{IB}= & \text { peso del órgano }(\mathrm{g}) \times 1000 / \text { peso cor- } \\
& \text { poral del ave }(\mathrm{g})
\end{aligned}
$$

El valor obtenido permite determinar el grado de atrofia de la bursa (Giambrone, 1987) donde:

$$
\begin{aligned}
& 1.5-3.5=\text { bursa normal } \\
& 0.5-1.5=\text { atrofia bursal } \\
& ? 0.5=\text { severa atrofia bursal }
\end{aligned}
$$

Para esta evaluación se eligieron 10 aves al azar por grupo a los 14, 21, 28, 35, 39 y 42 días de edad, las cuales fueron pesadas y posteriormente sacrificadas para la extracción de la Bursa de Fabricio. El tamaño se midió con el bursómetro (Falcón, 1996).

Los cambios histológicos se clasificaron de acuerdo al grado de severidad (Mohamed y Saif, (1996), donde:
$1=$ no hay lesiones

$2=$ leve depleción de células linfoides en pocos folículos $(30 \%)$

3 = moderada atrofia o depleción de células de los folículos (31-75\%), y

$4=$ severa necrosis y atrofia en todos los folículos (>75\%)

Para esta evaluación se tomaron al azar cinco muestras de bursa por grupo de las aves sacrificadas a los 28 días (antes del desafío) y a los 35 y 39 días de edad ( $3^{\circ}$ y $7^{\circ}$ día post-desafío, respectivamente). Se usó la tinción estándar de esosina-hematoxilina para el estudio histopatológico

\section{Mortalidad}

Se registró la mortalidad diaria y semanal, practicándose la necropsia para determinar las causas de muerte.

\section{Parámetros Productivos}

Se registró el peso corporal a todas las aves de cada grupo a $\operatorname{los} 7,14,21,28,35,42$ y 49 días de edad; así como el índice de conversión alimenticia (ICA) y el índice de eficiencia productiva (IEP) al final del estudio (Quintana, 1999)

\section{Evaluación Serológica}

Se tomaron muestras de sangre de 15 aves por grupo en el día 1, 32 y 49 de edad para medir el nivel de anticuerpos contra la enfermedad infecciosa de la bursa, mediante la prueba de ELISA de los laboratorios IDEXX.

\section{Análisis Estadístico}

Se utilizó el análisis de varianza para evaluar el peso corporal y los títulos de anticuerpos de los grupos experimentales. 


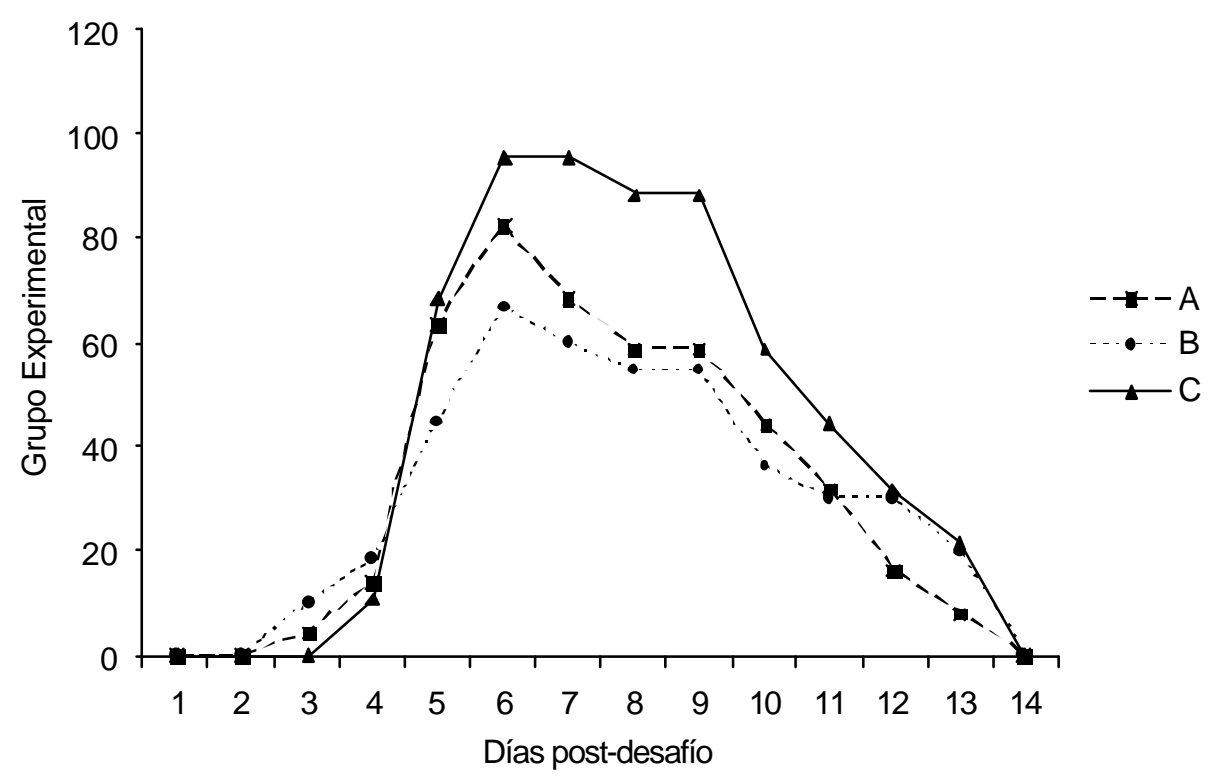

Figura 1. Ocurrencia de diarrea (\%) en pollos parrilleros $(n=321)$ sometidos a dos esquemas de vacunación contra la enfermedad infecciosa de la bursa (EIB) y que fueron desafiados con la cepa F52/70 de la EIB a los 32 días de edad.

A: Cepa 2512 (intermedia fuerte) a los 19 d; B: Cepa 2512 (intermedia-intermedia) a los 10 d y cepa 228 TC (intermedia-intermedia) a los $19 \mathrm{~d}$; C: grupo control no vacunado.

\section{Resultados}

Se presentó diarrea en todos los grupos a partir del $4^{\circ}$ día post-desafío, observándose el mayor porcentaje de aves afectadas en el $6^{\circ}$ y $7^{\circ}$ día post-desafío (Fig. 1)

Los valores de IB fueron disminuyendo a partir del $3^{\text {er }}$ día post-desafío; evidenciándose una atrofia bursal al $10^{\circ}$ día post-desafío en todos los grupos experimentales; especialmente en el grupo $\mathrm{C}$ no vacunado (Cuadro 1).

Al examen histopatológico se observó una depleción y atrofia linfoide moderada al $7^{\circ}$ día del desafio en el grupo A, así como una depleción y atrofia severa en los grupos B y C (Cuadro 2, Fig. 2).

Los pesos corporales después del desafío (35, 42 y 49 días de edad) fueron similares entre los 3 grupos experimentales, no encontrándose diferencia significativa ( $>>0.05$ ) entre ellos. Las aves del grupo B obtuvieron en promedio una ganancia de 276 g por encima del grupo $\mathrm{C}$, siendo el grupo $\mathrm{C}$ el que tuvo un mejor I.C.A. (Cuadro 2).

El promedio de los títulos de anticuerpos contra Gumboro, al final del experimento, fueron similares en los grupos vacunados (grupo A: 4167, CV: 20.3\%; grupo B: $3287, \mathrm{CV}$ : 38.9\%); grupo C: $1764, \mathrm{CV}: 55.1 \%$ ).

\section{Discusión}

La presentación de diarrea es uno de los principales signos clínicos de la EIB y se debe a una primera replicación del virus a nivel intestinal, dañando las células epiteliales de las vellosidades, aumentando así la permeabilidad en el intestino. La diarrea se observó desde el $4^{\circ}$ día del desafío en los 3 grupos experimentales, llegando a presentarse en el mayor número de aves al $6^{\circ}-7^{\circ}$ día del 
Cuadro 1. Índice bursal, tamaño bursal y grado de lesiones histológicas en pollos parrilleros $(\mathrm{n}=321)$ sometidos a dos esquemas de vacunación contra la enfermedad infecciosa de la bursa (EIB) y que fueron desafiados con la cepa F52/70 de la EIB a los 32 día s de edad

\begin{tabular}{ccccccc}
\hline & \multicolumn{7}{c}{ Edad (días) } \\
\cline { 2 - 7 } Grupo $^{1}$ & 14 & 21 & 28 & 35 & 39 & 42 \\
\hline Índice bursal $^{2}$ & & & & & & \\
$\mathrm{~A}$ & 2.16 & 2.28 & 2.45 & 2.36 & 1.58 & 1.42 \\
$\mathrm{~B}$ & 2.20 & 2.59 & 2.75 & 2.43 & 2.02 & 1.29 \\
$\mathrm{C}$ & 2.16 & 2.78 & 3.09 & 2.07 & 1.52 & 0.76
\end{tabular}

Tamaño bursal $(\mathrm{cm})$

$\begin{array}{lllllll}\text { A } & 4.3 & 6.0 & 6.1 & 7.2 & 6.8 & 5.8 \\ \text { B } & 4.8 & 5.8 & 6.4 & 7.2 & 7.2 & 5.8 \\ \text { C } & 4.3 & 5.3 & 6.7 & 6.3 & 6.0 & 4.8\end{array}$

Clasificación histológica ${ }^{3}$

$\begin{array}{llll}\text { A } & 3.0 & 2.6 & 3.0 \\ \text { B } & 2.0 & 3.0 & 3.6 \\ \text { C } & 3.0 & 3.0 & 4.0\end{array}$

1 A: cepa 2512 (intermedia fuerte) a los $19 \mathrm{~d}$; B: cepa 2512 (intermedia-intermedia) a los $10 \mathrm{~d}$ y cepa 228TC (intermedia-intermedia) a los $19 \mathrm{~d}$; y C: grupo control no vacunado

2 1.5-3.5 = normal; $0.5-1.5$ = atrofia bursal; $\leq 0.5$ = severa atrofia bursal

3 1: no hay lesiones; 2: leve depleción de células linfoides en pocos folículos (30\%); 3: moderada atrofia / depleción de células de los folículos (31-75\%); 4 : severa necrosis y atrofia en todos los folículos (>75\%).

Cuadro 2. Promedio de peso corporal, índice de conversión alimenticia (I.C.A.) e índice de eficiencia productiva (I.E.P.) a los 49 días de edad en pollos parrilleros $(n=321)$ sometidos a dos esquemas de vacunación contra la enfermedad infecciosa de la bursa (EIB) y que fueron desafiados con la cepa F52/70 de la EIB a los 32 días de edad

\begin{tabular}{cccc}
\hline Grupo $^{1}$ & Peso (g) & I.C.A. & I.E.P. \\
\hline A & 3,430 & 1.94 & 353 \\
B & 3,491 & 2.11 & 321 \\
C & 3,215 & 1.81 & 351 \\
\hline
\end{tabular}

1 A: cepa 2512 (intermedia fuerte) a los $19 \mathrm{~d}$; B: cepa 2512 (intermedia-intermedia) a los $10 \mathrm{~d}$ y cepa 228TC (intermedia-intermedia) a los $19 \mathrm{~d}$; y C: grupo control no vacunado 
(a)

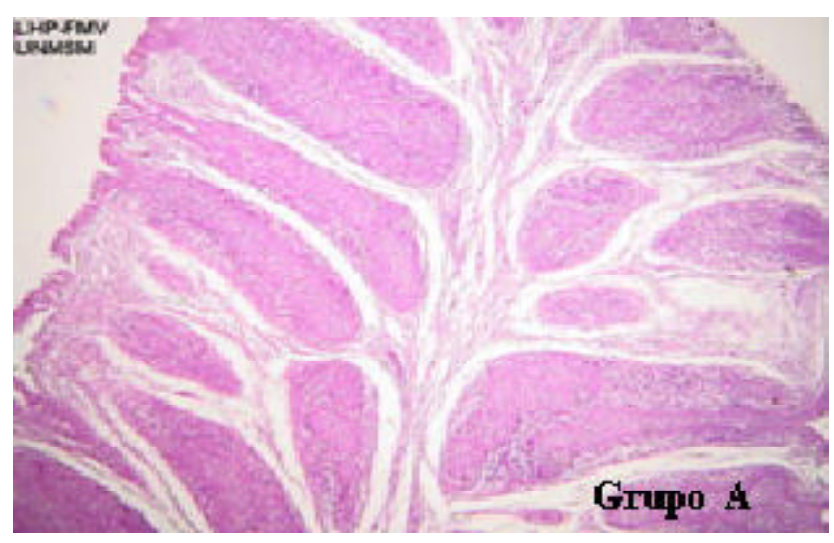

(b)

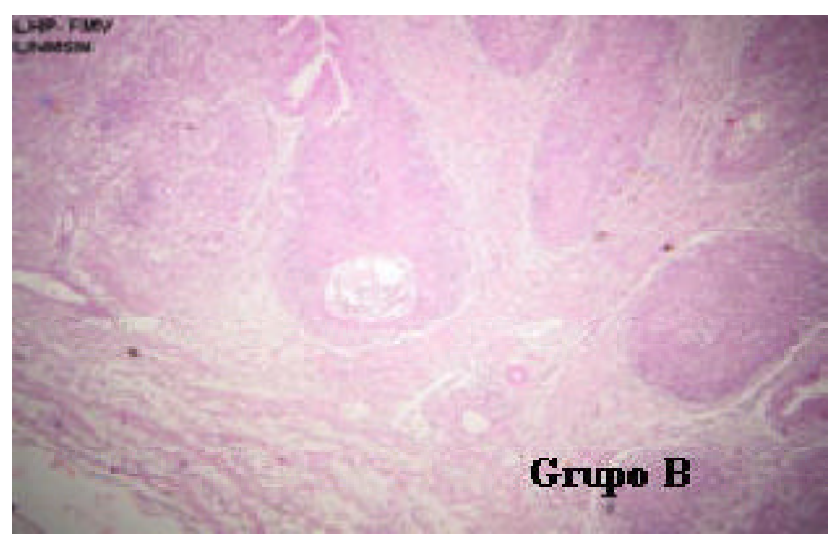

(c)

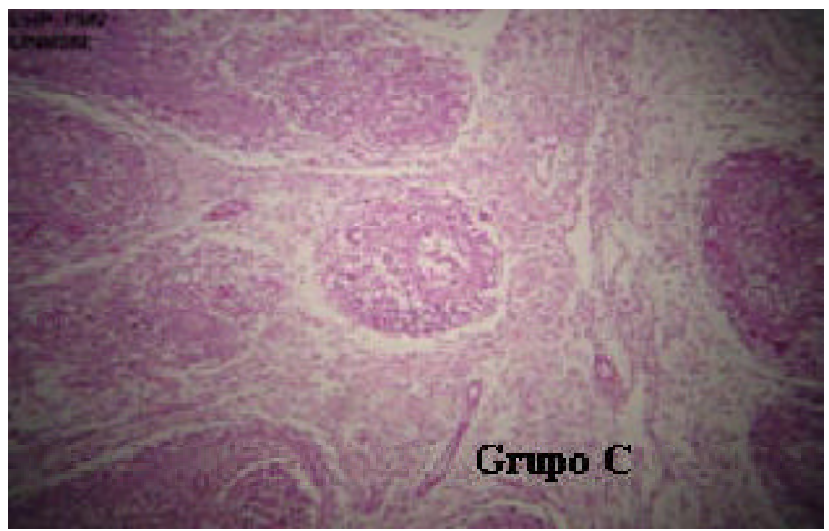

Figura 2. Grado de lesiones histológicas en pollos parrilleros al $7^{\circ}$ día del desafío con la cepa F52/70 y que fueran vacunados contra la enfermedad infecciosa de la bursa. (a) Bursa con una moderada depleción de linfocitos, en médula y corteza. (b) Se aprecia una fibroplasia interfolicular y una severa depleción de linfocitos en médula y corteza. (c) Presencia de algunos quistes foliculares, así como una severa depleción linfoide y fibroplasia interfolicular. 
desafío, especialmente en las aves del grupo no vacunado, donde se afectó hasta el $95 \%$ de las aves. Así mismo, todas las aves se llegaron a recuperar a las dos semanas del desafío.

Los resultados del tamaño de la bursa y el índice bursal indicaron que la cepa de desafío causó diversos grados de inflamación, edema y atrofia en las bursas de todos los grupos, lo cual fue corroborado con el examen histopatológico. Estos resultados discrepan con el estudio hecho por Ismail y Saif (1991), donde aves vacunadas con cepas en diferentes grados de atenuación y desafiadas con una cepa estándar STC no fueron afectadas, demostrando un buen nivel de protección con las vacunas usadas.

Las lesiones histopatológicas de los grupos A y B coinciden con otros estudios (Lucio y Hitchener, 1979; Winterfield et al., 1981; Casaubon et al., 1993; Guerrero et al., 1993; Sánchez et al., 1993) donde se reportan lesiones de mediana a intermedia intensidad causado al tejido bursal como consecuencia de la replicación del virus vacunal usado y cuyo grado de severidad es dependiente de los anticuerpos maternales de las aves.

Así mismo, el grado de lesión de las bursas se puede atribuir a la patogenicidad y antigenicidad de las cepas vacunales, lo que ayuda a tener una mejor protección contra la multiplicación del virus de desafío, sin causar signos de la enfermedad ni afectar los parámetros productivos de las aves.

Los resultados de serología muestran que a los 32 días de edad, día del desafío, todas las aves mostraban niveles bajos de anticuerpos, debido a que los anticuerpos maternales fueron disminuyendo durante las primeras semanas de edad y aun no se evidenciaba la seroconversión de los grupos vacunados, además que no estuvieron expuestos a un antígeno viral. A los 17 días del desafío se produjo una elevación de los títulos de anticuerpos en todos los grupos, especialmente en los grupos vacunados, debido a que estos grupos fueron previamente sensibilizados, de allí que las cepas vacunales estimularon las células de memoria. Esta respuesta concuerda con investigaciones hechas por Mohamed y Saif (1996). Sin embargo, estos resultados difieren con estudios realizados para otros agentes, como la enfermedad de Newcastle y Bronquitis Infecciosa, donde el grupo no vacunado es el que presenta el mayor nivel de seroconversión debido a una elevada respuesta activa frente a una primo infección.

La ausencia de mortalidad por Gumboro después del desafío en los tres grupos experimentales, está relacionado a la patogenicidad de la cepa de desafío (Rautenschlein et al., 2000).

Los grupos vacunados mostraron una buena protección frente al desafío ya que los parámetros productivos no fueron afectados. Estos resultados concuerdan con estudios realizados en Venezuela y Holanda, donde se aplicaron vacunas "calientes" contra la EIB que fueron capaces de controlar virus de campo (Gardin, 2003).

\section{Conclusiones}

Los dos programas de vacunación confirieron una buena protección ante el desafío con una cepa estándar de la enfermedad de Gumboro.

\section{Literatura Citada}

1. Banda A, Villegas P. 2002. Nuevas técnicas para el diagnóstico y tipificación de cepas de campo del virus de la enfermedad de Gumboro. En: Evolución de la enfermedad de Gumboro e innovaciones para su control. XIV Curso de Actualización Avimex. México. p 19-27. 
2. Casaubon M, Altamirano $R$, Valladares J. 1993. Estudio clínico del sistema inmunocompetente en tres parvadas de pollo de engorda. En: IV Jornada Médico Avícola. México D.F.: Universidad Nacional Autónoma de Mexico. p 50-63.

3. Falcón L. 1996. Efecto de dos programas de vacunación para infección bursal en la presentación de hepatitis a cuerpos de inclusión en pollos de carne. Tesis de Médico Veterinario. Lima: Facultad de Medicina Veterinaria, Univ. Nacional Mayor de San Marcos. 54 p.

4. Gallardo WL. 1998. Evaluación serológica e histopatológica de pollos broilers vacunados contra la enfermedad de Gumboro a los doce días de edad. Tesis de Médico Veterinario. Lima: Facultad de Medicina Veterinaria, Univ. Nacional Mayor de San Marcos. 36 p.

5. Gardin Y. 2003. Enfermedad de Gumboro muy virulenta en Latinoamérica: Puntos importantes y consecuencias prácticas. En: XVII Congreso Latinoamericano de Avicultura. Bolivia. p 14-18.

6. Guerrero D. 2001. Evaluación de la patogenicidad de las cepas vacunales intermedias S-706 y 2512 del virus de Gumboro en la bursa de Fabricio en pollos de carne. Tesis de Médico Veterinario. Lima: Facultad de Medicina Veterinaria, Univ. Nacional Mayor de San Marcos. 51p.

7. Guerrero $R$, Valladares de la Cruz $C$, Quintana J. 1993. Evaluación del daño bursal mediante el índice bursal, rango tarso bursal y el estudio histopatológico en pollos de engorde vacunados contra la infección de la bolsa de Fabricio. En: IV Jornada Médico Avícola. México DF. p 93-98.

8. Giambrone J. 1987. Evaluación y relaciones morfométricas en la enfermedad infecciosa de la bursa como método de diagnóstico. Georgia, USA: The American of Avian Pathology. p 24-38.

9. Hafez H. 2002. Infección de la bolsa de Fabricio. En: Evolución de la enfermedad de Gumboro e innovaciones para su control. XVI. Curso de Actualización México: Avimex. p 7-15.

10. Ismail N, Saif Y. 1991. Inmunogenicity of infectious bursal disease viruses in chickens. Avian Dis 35: 460-469.

11. Lucio B, Hitchner S. 1979. Adaptation and antigenic variation of infectious bursal disease virus. J Chin Soc Vet Sci 12: 297304.

12. Mohamed K, Saif Y. 1996. Influence of the host system on the pathogenicity, immunogenicity, and antigenicity of infectious bursal disease virus. Avian Dis 40: 553-561.

13. Quintana J. 1999. Avitecnia: Manejo de las aves domésticas. $3^{\circ}$ ed. México: Ed. Trillas. 384 p.

14. Rautenschlein S, Hung-Yueh Y, Sharma J. 2000. The effect of T cell depletion on vaccine protection against infectious bursal disease. In: Proc. XLIV Western Poultry Disease Conference. Sacramento, California, USA.

15. Saif Y. 2003. Vacunas y vacunación contra la enfermedad de Gumboro. Mundo Avícola y Porcino 43: 7-8.

16. Sánchez C, Casaubon M, Valladares de la Cruz J, Tapia G. 1993. Evaluación de dos cepas vacunales del virus de la infección de la bolsa de Fabricio (IBF) en pollos de engorde comerciales. En: IV Jornada Médico Avícola. México DF. p 34-39.

17. Winterfield R, Dhillon A, Thacker $H$. 1981. Characteristics of apparent derivatives of the 2512 strain of infectious bursal disease virus when used as vaccines. Avian Dis 25: 900-910. 